\title{
THE RADIATION HYPOTHESIS OF CHEMICAL REACTIVITY AND SOME OF ITS APPLICATIONS.
}

\author{
By W. C. McC. Lewis, M.A., D.Sc., Brunner Professor of \\ Physical Chemistry in the University of Liverpool.
}

The term catalysis as usually employed is a comprehensive one, including. non-stoicheiometric effects as well as stoicheiometric. As examples of the latter we have reactions brought about by ions and molecules of solutes in homogeneous solutions: non-stoicheiometric catalysis being found in the influence of the solvent in the case of homogeneous solutions and by the surface of the material in heterogeneous systems.

The intermediate compound view of catalysis, which has been very generally accepted and for which there is considerable experimental evidence, is not a general theory of catalysis as a whole. It applies essentially to stoicheiometric catalysis with a possible extension to certain cases of heterogeneous catalysis in which it is difficult and perhaps unnecessary to. draw a sharp line between adsorption and temporary chemical combination.

In the case of homogeneous systems the intermediate compound view affords an explanation of one aspect of catalysis, in so far, in fact, as the material mechanism of a process is concerned. It serves in short to bring catalytic reactions into precisely the same category as ordinary stoicheiometric processes to which we would not apply the term catalytic. It cannot do more than this. The further theoretical treatment of stoicheiometric catalysis becomes identical with the treatment which must be applied to non-catalytic processes.

The characteristic feature of positive catalysis is the usually enormous increase in velocity brought about by the presence of the catalyst. On the basis of the intermediate compound theory this is due to the formation of certain entities which, in the absence of the catalyst, are either not produced at all or at most in minute amounts. The increase in speed is directly attributed to the greatly increased concentration of reacting individuals. Whilst this is undoubtedly correct for stoicheiometric catalysis no similar treatment seems possible for non-stoicheiometric catalysis. The explanation of the latter type of catalysis cannot be found in material considerations alone; it is necessary to consider the energy changes which are involved as a preliminary to the material changes observed.

That absorption of radiation is the source of chemical change in general was pointed out several years ago by Professor Perrin. In particular Professor Perrin offered the first (qualitative) explanation of the mechanism of uni-molecular processes. ${ }^{l}$ The same mode of treatment was independently applied to catalysis by the writer in $1914^{2}$ thereby extending the significance

1 "Les Atomes," Igr3.

${ }^{2}$ Cf: Fourn. Chem. Soc., 105, 2330 (I914); ibid. 107, 233 (I915). 


\section{RADIATION HYPOTHESIS OF CHEMICAL REACTIVITY}

of Marcelin's concept of the critical energy to reactions whether catalytic or non-catalytic. By introducing the Quantum Theory it is possible to deduce not only the equation of Arrhenius but also the Einstein law of the photochemical equivalent in a very simple manner.

On the radiation basis it can be shown ${ }^{1}$ that the rate of a reaction depends not only on the concentration of the reacting substances, as the law of mass action requires, but also on a dimensionless exponential term, $e^{-\mathrm{E} / \mathrm{RT}}$, where $\mathrm{E}$ is the critical increment or energy of activation per grammolecule. The greater the value of $\mathrm{E}$ the smaller is the exponential, i.e. the smaller the rate, and, further, a small change in $\mathrm{E}$ produces a marked effect on the velocity. It is obviously the exponential term which leads to the deduction of the Arrhenius expression for the influence of temperature on the velocity constant. Considering the opposed reaction as well and introducing the van't Hoff isochore we obtain at once the relation: heat evolved $=\mathrm{E}$ resultant $-\mathrm{E}$ reactant.

The suggestion is made that in non-stoicheiometric catalysis (solvent catalysis) the alterations in the velocity observed are due essentially to alterations in the value of $\mathrm{E}$; in stoicheiometric processes the increase in the rate with increase in the concentration of the catalyst is due not to a .change in $\mathrm{E}$ but to a change in the concentration term upon which the exponential is multiplied. (Naturally if so much solute catalyst is employed that the nature of the medium alters there will likewise be an alteration in $\mathrm{E}$, since in such a case we are superimposing a solvent effect upon the stoicheiometric catalytic process.)

That stoicheiometric catalysis, e.g. catalysis by means of ions, involves no sensible change in $\mathrm{E}$ is shown by the results obtained in the hydrolysis of esters and the inversion of sucrose by hydrochloric acid.

The following table contains the data for the velocity constant and the temperature coefficient in the latter case. It cannot be claimed that the catalytic conditions (as determined by the activity of the hydrogen ions) are identical at the two temperatures, but they are approximately so.

INVERSION OF SUCROSE (2O PER CENT. SOLUTION).

\begin{tabular}{|c|c|c|c|c|c|}
\hline \multirow{2}{*}{$\begin{array}{c}\text { Concentration of } \\
\mathrm{HCl} \text {. }\end{array}$} & \multicolumn{3}{|c|}{ Mean Velocity Constant $\times$ ro $^{3}$} & \multirow{2}{*}{$k_{35} / k_{25}$. } & \multirow{2}{*}{$k_{45} / k_{35}}$. \\
\hline & At $25^{\circ}$ & At $35^{\circ}$. & At $45^{\circ}$. & & \\
\hline $0.0502 \mathrm{~N}$ & 0.4169 & $x \cdot 738$ & $6 \cdot 213$ & $4^{\cdot 1} 7$ & 3.57 \\
\hline 0.2512 & $2 \cdot 255$ & $9: 355$ & $35.86^{\circ}$ & $4^{\cdot} I_{4}$ & $3 \cdot 83$ \\
\hline 0.4137 & 4.043 & I $77^{\circ} \mathrm{OO}$ & $60 \cdot 62$ & $4^{\cdot 20}$ & 3.57 \\
\hline $0^{\circ}$ goo & II $=16$ & $46 \cdot 76$ & $\mathrm{r}_{4} 8 \cdot 8$ & $4 \cdot 99$ & $3 \cdot 18$ \\
\hline$I \cdot 2 I_{4}$ & $I_{7} \cdot 45$ & $75^{\circ} 97$ & - & 4.35 & - \\
\hline
\end{tabular}

The variations in the value of the temperature coefficient over a given temperature range are irregular and not very large; at the same time the velocity constant increases over fortyfold. It is evident that the increase in the velocity is not due to any sensible alteration in $\mathrm{E}$ (which is calculated from the temperature coefficient), but to the production of higher concentrations of reactants possessing approximately a constant critical increment. 
It is of interest to contrast the relative independence of the value of $\mathrm{E}$ in respect of the concentration of a solute catalyst, such as hydrogen ion, acting stoicheiometrically, with the large variation in $\mathrm{E}$ produced by altering the nature of the solvent (non-stoicheiometric catalysis). The data of von Halban $^{1}$ on the decomposition of triethylsulphine bromide in various solvents afford a good example of the effect as is shown in the following table :--

DECOMPOSITION OF TRIETHYLSULPHINE BROMIDE.

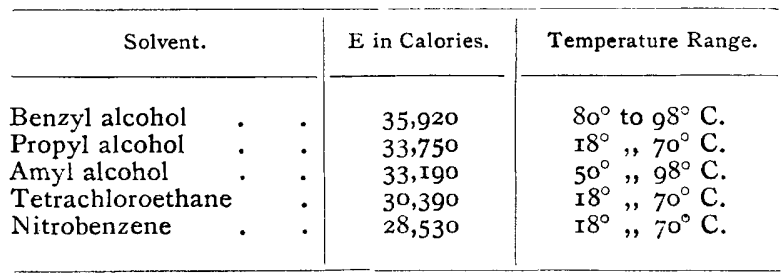

Analogous results have been obtained by Dimroth ${ }^{2}$ for the decomposition of the methyl ester of benzyl-5-hydroxy-I.2.3. triazole carboxylic acid :-

\begin{tabular}{|c|c|c|c|}
\hline \multicolumn{2}{|c|}{ Solvent. } & $E$ in Calories. & Temperature Range. \\
\hline $\begin{array}{l}\text { Chloroform } \\
\text { Ethyl alcohol } \\
\text { Acetone }\end{array}$ & $\begin{array}{l}\cdot \\
\dot{*}\end{array}$ & $\begin{array}{l}35,420 \\
30,640 \\
29,140\end{array}$ & $\begin{array}{l}40^{\circ} \text { to } 50^{\circ} \mathrm{C} . \\
40^{\circ}, 50^{\circ} \mathrm{C} . \\
40^{\circ}, 50^{\circ} \mathrm{C} .\end{array}$ \\
\hline
\end{tabular}

Further the measurements of $\mathrm{Cox}^{3}$ show a similar effect in the case of a simple addition reaction, namely the addition of aniline to bromoacetophenone :-

\begin{tabular}{|c|c|c|c|}
\hline Solver & & & E in Calories. \\
\hline $\begin{array}{l}\text { Benzene } \\
\text { Chloroform : } \\
\text { Nitrobenzene } \\
\text { Ethyl alcohol } \\
\text { Benzyl alcohol }\end{array}$ & : & $\begin{array}{ll}\cdot & \cdot \\
\cdot & \cdot \\
\dot{*} & \cdot \\
\cdot & \cdot \\
\cdot & \cdot\end{array}$ & $\begin{array}{r}8,088 \\
10,760 \\
13,470 \\
I_{3,910} \\
I_{4,290}\end{array}$ \\
\hline
\end{tabular}

That the effect of different solvents is not stoicheiometric is perhaps most directly shown by considering the alteration produced in the velocity of a reaction by changing the composition of a mixed solvent. Thus, in a mixture of sulphuric acid and water, containing $94^{\circ} 6$ per cent. acid the value of $\mathrm{E}$ for the transformation of acetophenoneoxime into acetanilide ${ }^{4}$ is 21,720 calories per grammolecule, whilst for a mixture containing 93.6 per

\footnotetext{
${ }^{1}$ Zeitsch. physikal. Chem., 77, r29 (rgog).

3 Fourn. Chem. Soc., rrg, I42 (I92I).

${ }^{2}$ Lieb. Ann., 373, 336 (19ro).

${ }^{4}$ Cf. Lobry de Bruyn and Sluiter, Proc. K. Akad. Wetenschap. Amsterdam, 6, 773
} (1904). 
cent. acid the value of $\mathrm{E}$ is $24,670 \mathrm{cals}$. In general the effect produced on the velocity of the reaction is an exponential function of the concentration of one of the components of the mixed solvent, a relationship first discovered by Rosanoff. ${ }^{1}$ Compare also Jones and Lewis. ${ }^{2}$

Whilst emphasising this influence of the solvent on the value of $\mathrm{E}$ it is not intended to convey the impression that addition compounds involving the solvent are absent. To simply postulate their existence, however, cannot be a satisfactory explanation of solvent catalysis.

Instead of making the somewhat cumbrous distinction between stoicheiometric and non-stoicheiometric effects we may state the matter thus :-

Intermediate compounds are formed in general; their nature and stability, as measured by their critical increments or energy of activation, vary with the nature of the solvent, being determined by the electromagnetic properties (essentially the vibration frequencies of the valency electrons) of the solvent and solute concerned.

The most fundamental problem involved therefore in catalytic as well as non-catalytic processes is the mode whereby the energy necessary for the chemical change is communicated to the reactant unit. It is in this connection that recourse has been had to the radiation hypothesis of chemical reactivity.

According to this hypothesis, in the case of thermal processes, the radiation which is necessarily present in virtue of the temperature of the system and which possesses a certain density for each wave-length region, furnishes the energy which is required for the chemical change. The absorption is considered to be continuous. This energy may either be communicated in terms of a single quantum corresponding to a given frequency or in terms of a small number of quanta of correspondingly lower frequency. This point is considered in some detail later. It is important to observe that we are considering at the moment a single chemical process, such as the dissociation of a diatomic gas. This case is clearly different from that met with in solutions in which a complex molecule is ruptured at more than one position. In this case the total increment is necessarily made up of a number of smaller increments.

Under thermal conditions the act of absorption diminishes the density of the radiation thus removed; and this is made good by all the other types of radiation present in accordance with the view of Planck regarding the constitution and maintenance of temperature radiation.

In virtue of this redistribution of radiant energy among the different wave-lengths the temperature of the system tends to fall. In the majority of reactions, however, by means of a thermostat, it is possible to maintain the average temperature of the system, so that we can speak of an isothermal process and velocity constant. It is evident that in such reactions the rate of adjustment of the radiation density is more rapid than the rate at which a certain type of radiation is being removed. It is this kind of reaction which is usually considered.

One of the most striking conclusions which can be drawn from the application of the radiation hypothesis to solute catalysis in a homogeneous system is that the equilibrium constant of the process is in general influenced by the catalyst. ${ }^{3}$ It is only when the heat of the reaction is zero or nearly so that the equilibrium point remains unaffected. This conclusion is in 
agreement with observations made in connection with the hydrolysis of esters and esterification of acids.

The radiation hypothesis, based as it is upon the quantum theory of Planck, harmonises with views on atomic and molecular structure in which the quantum theory plays a fundamental part. Since Bohr in I9I3 put forward his now familiar theory of atomic structure the concept of a given atom or molecule existing in a number of steady states which differ from one another in respect of energy has come to be generally accepted even by those who do not regard the particular distribution and modes of motion of the electrons as postulated by Bohr to be necessarily correct.

The significance of such stages from the point of view of chemical change has been emphasised in particular by E. C. C. Baly. ${ }^{1}$ It is obvious that different degrees of activation are required for different chemical changes of one and the same reactant. A good example of this is furnished by the ozone molecule, $\mathrm{O}_{3}$.

In the first place ozone can be decomposed thermally at a measurable rate at relatively low temperatures such as $100^{\circ} \mathrm{C}$. Under these conditions it has been shown by Warburg, ${ }^{2}$ by Clement ${ }^{3}$ and by Chapman and Jones ${ }^{4}$ that the decomposition is bimolecular. Applying the equation for bimolecular reaction velocities employed by the writer in other cases, ${ }^{5}$ it is possible to calculate the value of the exponential term $e^{-2 \mathrm{E} / \mathrm{RT}}$, which appears in the velocity expression, by making use of the data of Chapman and Jones. The details of the calculation will be given elsewhere. It is found that $\mathrm{E}=\mathrm{I} \mathrm{I}, 800$ cals. The accuracy of this quantity depends mainly on the accuracy of the value adopted for the range of approach in a collision between two ozone molecules. This value of $\mathrm{E}$ agrees satisfactorily with that experimentally determined by Clement, namely, $2 \mathrm{E}=26,000$ or $\mathrm{E}=$ $x_{3,000}$ cals. E represents the amount of energy which must be supplied to one grammolecule of ozone, over and above its average energy content, to enable such a molecule to react in a bimolecular manner with another similarly activated. It represents a low partial activation of the ozone molecule. The wave-length corresponding to $E=13,000$ is $2 \cdot 17 \mu$, that corresponding to $\mathrm{E}=\mathrm{II}, 800$ being $2.40 \mu$; that is the activation corresponds to the short infra-red region. It is worthy of note that according to Warburg and Leithauser ${ }^{6}$ ozone possesses a characteristic absorption band in the short infra-red at $\lambda=4756 \mu$. This is approximately double the wave-length or half the frequency obtained from a consideration of Chapman and Jones' results, indicating probably that two quanta of the $4.756 \mu$ type are involved in the low partial activation of the ozone molecule necessary to allow of bimolecular decomposition.

Higher stages of partial activation of the $\mathrm{O}_{3}$ molecule are indicated by absorption bands in the visible region. Liveing and Dewar ${ }^{7}$ have observed four such bands, namely, at the wave-lengths $602 \mu \mu, 57 \mathrm{I} \mu \mu, 532 \mu \mu$, and $5 \circ 3 \mu \mu$. Of these the first and last are clearly distinct from the oxygen bands observed by the same investigators. The same may be true of the two intermediate values recorded, though it is less certain. Chappuis had previously observed a band at $628 \mu \mu$. In the near ultra-violet, numerous bands have been observed by Fowler and Strutt. ${ }^{8}$

In the further ultra-violet region, ozone is characterised by a band with

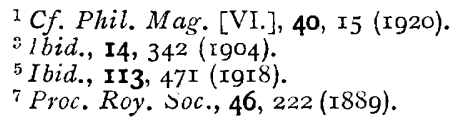

5 Ibid., I4, 342 (r9o4).

${ }^{7}$ Proc. Roy. Soc., 46, 222 (1889).

${ }^{2}$ Ann. Physik [IV.], 9, I286 (I902).

${ }^{4}$ Fourn. Chem. Soc., 97, 2463 (Igro).

¿Ann. Physik, [IV.], 28, 31 (I909).

${ }^{8}$ Ibid., 93 A, 577 (IgI7.) 


\section{RADIATION HYPOTHESIS OF CHEMICAL REACTIVITY}

its head at $254 \mu \mu .^{1}$ Ozone is decomposed at this wave-length, but, for reasons to be given below, it is concluded that this does not correspond to the lowest possible energy required for unimolecular decomposition of ozone. Ozone should also possess a band in the extreme ultra-violet corresponding to the dispersional period. So far as the writer is aware nothing is known on this point.

In addition to the thermal bimolecular decomposition, ozone is likewise known to decompose photochemically, which we may take to be a unimolecular process, usually represented by the stoicheiometric equation :

$$
\mathrm{O}_{3} \rightarrow \mathrm{O}_{2}+\mathrm{O} \quad . \quad . \quad . \quad . \quad \text { ( }
$$

We have now to consider the formation of ozone from oxygen. As ordinarily formed by radiation from a quartz mercury lamp the process is presumably equation ( $\mathrm{r}$ ) reversed. On this basis the process requiring the greatest absorption of energy is the act of dissociating molecular oxygen into free atoms. Siegel ${ }^{2}$ estimated the energy required as 160,000 cals. per grammolecule. This is little more than guess-work, but that it is of the right order of magnitude is shown by the fact that the wave-length corresponding with this energy is $\mathrm{I} 80 \mu \mu$ which would be in agreement with the conclusion arrived at by Lyman, ${ }^{3}$ that the radiation capable of forming ozone is greatly absorbed by quartz and completely absorbed by $\mathrm{I} \mathrm{mm}$. of air. Steubing ${ }^{4}$ concludes that $\lambda=\mathrm{r} 85 \mu \mu$ is capable of dissociating oxygen, that is $E_{O}=151,000$ cals. per grammolecule. The investigation of L. and E. Bloch ${ }^{5}$ also indicates bands at wave-lengths in the neighbourhood of I80 to $190 \mu \mu$. As a rough approximation, therefore, we may take $\mathrm{E}_{\mathrm{O}_{2}}={ }_{1} 5 \mathrm{I}, 000$ cals.

The heat of formation of ozone per grammolecule is 35,000 cals. absorbed, the weighted mean of results obtained by Muellen ${ }^{6}$ and Kailan and Jahn. ${ }^{7}$ This refers to the formation of ozone from molecular oxygen. The relation to be applied is therefore :- heat evolved $=$ critical increment of resultant - critical increment of reactants, which may be written in this case as

$$
-35000=\mathrm{E}_{\mathrm{O}_{3}}-\frac{1}{2} \mathrm{E}_{\mathrm{O}_{2}}
$$

where $\mathrm{E}_{\mathrm{O}_{3}}$ is the critical increment of dissociation of the ozone molecule as represented by equation ( $\mathrm{I}$ ) above, and $\mathrm{E}_{\mathrm{O} . \mathrm{z}}$ is the energy absorbed in the dissociation of the oxygen molecule into free atoms. In writing the process in this way it is assumed that the molecule of oxygen does not require any sensible activation in order to react with a nascent oxygen atom. We thus find-

$$
\mathrm{E}_{\mathrm{O} 3}=\frac{1}{2} \mathrm{E}_{\mathrm{O} 2}-35000=\frac{\mathrm{I} 5 \mathrm{I000}}{2}-35000=40500 \text { cals. }
$$

This energy corresponds to a frequency $4.29 \times 10^{14}$ or $\lambda=700 \mu \mu$. In view of the possible error in the value taken for the energy of dissociation of oxygen, we are only justified in concluding that on the above basis ozone should be decomposed in a unimolecular manner by radiation in the visible region of the spectrum, probably at a wave-length greater than $500 \mu \mu$.

${ }^{1}$ Kruger and Moelle, Physikal. Zeitsch., 13, 728 (Igr2); Fabry and Buisson, Fourn. de Physique, 3, Ig6 (I9r3).

${ }^{2}$ Zeitsch. physikal. Cuem., 87. 64 I (Ior4).

3 "Spectroscopy of the Extreme Uitra-violet," p. 67.

4 Ann. Physik [IV.] 33, 555 (rgro).

${ }^{6}$ Rec. Trav. Chim. Pays-Bas., 2, 99 (1883).

7 Zeitsch. anorg. Chem., 68, 243 (1910). 
This conclusion was arrived at by the writer in r9r8, but only within the present year has it been possible to carry out the necessary experimental work in order to test it. The results obtained in the writer's laboratory by Mr. R. O. Griffith and Mr. W. J. Shutt (as yet unpublished) completely support the view that ozone can be decomposed by light in this region, decomposition of ozone being effected by light transmitted by a red filter, that is by wave-length longer than $670 \mu \mu$.

The above result is somewhat surprising in that ozone is usually regarded as being decomposed unimolecularly in the ultra-violet at $\lambda=254 \mu \mu$. It follows that the latter wave-length must correspond to a process of a more violent character than that represented by equation ( $\mathrm{I}$ ).

Of the possibilities which present themselves we may consider in the first place the process: $\mathrm{O}_{3} \rightarrow 3 \mathrm{O}$

Applying the quantum relation to this process we have :-

$$
\text { heat evolved }=\frac{3}{2} \mathrm{E}_{\mathrm{O}_{2}}-\mathrm{E}_{\mathrm{O}_{3}}
$$

where $\mathrm{E}_{\mathrm{O}_{2}}$ stands as before for the energy of dissociation of oxygen per grammolecule ( $c a$ I 5 I,000 cals.) and $\mathrm{E}_{\mathrm{O} 3}=\mathrm{III}, 000$ cals., the energy corresponding to $\lambda=254 \mu \mu$. It follows on this basis that the heat evolved would be + I 5,000 cals., whilst the observed value is $+35,000$ cals. The discrepancy is so great that we may dismiss the possibility that $\lambda=254 \mu \mu$ causes decomposition of ozone simply into free oxygen atoms.

Another possibility is that the decomposition of ozone in the ultra-violet gives rise to electrically charged resultants which further react with one another so as to give finally electrically neutral molecular oxygen. The suggestion that ozone decomposes at $254 \mu \mu$ into electrically charged individuals has been made by Rideal and $\mathrm{Kun} z,{ }^{1}$ who have attempted to deal quantitatively with the various energy terms which are involved. They make use, however, of the value $9^{*} 2$ volts for the ionisation potential of oxygen, the accepted value at the time. The subsequent measurements of Mohler and Foote ${ }^{2}$ show that the ionisation potential of this gas is I $5.5 \pm 0.5$ volt. (in excellent agreement with the value 15.2 volts, calculated from the dispersional frequency), whilst there is also a resonance potential at $7.9 \mathrm{I} \pm 0^{\circ} \mathrm{I}$ volt which corresponds with the wave-length ${ }_{5} 7 \mu \mu$, which is almost certainly, therefore, the head of the well-known broad band in the far ultra-violet in oxygen. Rideal and Kunz state that they hope to test experimentally whether electrically charged particles are formed by the decomposition of $\mathrm{O}_{3}$ by light of wave-length $254 \mu \mu$.

Incidentally it may be shown with some degree of certainty that the oxygen band with its head at I $57 \mu \mu$ is not that which accounts for the photochemical formation of ozone by the quartz mercury lamp. ${ }^{3}$ If this were the case, then, the resonance potential at $7.9 \mathrm{I}$ volts would correspond with the energy of dissociation of oxygen into normal atoms; that is $\mathrm{E}_{\mathrm{O}_{2}}$ would be $18 \mathrm{r}, 000$ cals. and consequently on making use of the expression :

$$
\mathrm{E}_{\mathrm{O} 3}=\frac{1}{2} \mathrm{E}_{\mathrm{O}_{2}}-35,000 \text {, }
$$

we would obtain $\mathrm{E}_{\mathrm{O}_{3}}=55,500$ cals., which would correspond to $\lambda=508 \mu \mu$,

1 Fourn. Physical Chem., 24, 379 (I920).

${ }^{2}$ Bull. Bur. Standards, Scientific Paper No. 400 (I0,20).

${ }^{3}$ Light of wave-length ${ }_{57} \mu \mu$ could not be transmitted by quartz. This, however, is not in itself definitive in the present case, as oxygen is capable of absorbing over a characteristically wide range, with the result that a band having its head at $157 \mu \mu$ may extend into the region which quart $z$ could just transmit. For the purpose o applying the quantum heat relation, however, it is necessary to carry out calculation of energy quantities in terms of the head of a band. 
as the longest wave-length of light capable of decomposing ozone in a unimolecular manner. Griffith and Shutt have found, however, that ozone can be decomposed by light in the visible region of wave-length longer than $670 \mu \mu$.

There is a further possibility in connection with the decomposition of ozone by $\lambda=254 \mu \mu$, namely, the production of an electrically neutral molecule and atom of oxygen each of which is activated in the sense that at least one of the electrons is displaced to an orbit outside that characteristic of the normal atom and molecule. A similar concept has been applied in other cases, e.g. in the case of hydrogen by Franck, Knipping and Kruger. ${ }^{1}$ We may attempt a semi-quantitative treatment of this possibility in the following way:-

We begin by assuming as before that the process: $\mathrm{O}_{2} \rightarrow 2 \mathrm{O}$, in which the oxygen atoms are normal, requires an absorption of $\mathrm{I}_{5} \mathrm{r}, 000$ cals. per grammolecule. Secondly, the process which corresponds to the resonance potential, $7.9 \mathrm{I}$ volts (i.e. $\mathrm{E}=\mathrm{I} 8 \mathrm{I}, 000$ cals.), may be reasonably ascribed to dissociation into atoms at least one of which has a displaced electron and is therefore activated above the normal, thus :

$$
\mathrm{O}_{2} \rightarrow \mathrm{O} \text { normal }+\mathrm{O} \text { active. }
$$

On combining the two expressions we obtain :

$\mathrm{O}$ normal $\rightarrow \mathrm{O}$ active with the absorption of 30,000 cals.

As regards the partial activation of the oxygen molecule Liveing and Dewar ${ }^{2}$ lay stress on the persistence of the band at $\lambda=765 \mu \mu$. This necessarily represents a stage of partial activation of the molecule, and it may be the one produced in the present case when ozone is decomposed by ultra-violet light. The $\mathrm{E}$ value corresponding to $765 \mu \mu$ is 37,000 cals. Employing this value we can write on the basis of the suggestion made above for the decomposition of ozone by $\lambda=254 \mu \mu$ :

$$
\mathrm{O}_{3} \rightarrow \mathrm{O}_{2} \text { active }+\mathrm{O} \text { active. }
$$

The quantum expression for the heat effect is then:

$$
\begin{aligned}
35000 & =\mathrm{E}_{\mathrm{O}_{2}} \text { active }+\mathrm{E}_{\mathrm{O}} \text { active }-\mathrm{E}_{\mathrm{O}_{3}} \\
& =37000+\left(\frac{\mathrm{I}_{5} \mathrm{I000}}{2}+30000\right)-\mathrm{E}_{\mathrm{O}_{3}} \\
& =37000+\mathrm{r} 05500-\mathrm{E}_{\mathrm{O}_{3}} . \\
\text { whence, } \mathrm{E}_{\mathrm{O} 3} & =107,500 \text { cals. }
\end{aligned}
$$

The value of $\mathrm{E}_{\mathrm{O} 3}$ corresponding to $\lambda=254 \mu \mu$ is I I I,000 cals., which is in fair agreement with the value calculated on the above basis.

Such considerations as the foregoing serve to illustrate the mode of treatment which the application of the quantum theory permits when reactions are viewed from that standpoint. It is evident that further progress. in this direction in connection with gaseous reactions depends on further experimental measurements of photochemically effective wave-lengths, absorption bands, and resonance and ionisation potentials.

Turning to other problems, brief mention may be made of the fact that, in the case of reactions in solution to which the concept of energy of activation and the radiation hypothesis have been applied, the first direct proof that the solute, which is undergoing change, exhibits a characteristic absorption band, the position of which is in quantitative agreement with that calculated on the radiation hypothesis, has recently been obtained by $\mathrm{Mr}$. 
H. A. Taylor in the writer's laboratory. The reaction referred to is the decomposition of triethylsulphine bromide in nitrobenzene, investigated by von Halban, ${ }^{1}$ from whose data the critical increment $\mathrm{E}$ is found to be $28,53^{\circ}$ cals. corresponding to the frequency $3^{\circ} \circ \times 10^{14}$ or wave-length $\mathrm{r} \cdot 0 \mu$. Using a Hilger infra-red spectrometer and a radiomicrometer, Mr. Taylor has examined the absorption spectrum of triethylsulphine bromide in nitrobenzene over the range $0.8 \mu$ to $3^{\cdot} I \mu$, and has found that the bromide exhibits only one distinct band in this range, namely at $\mathrm{I} \cdot 05 \mu$, which agrees very satisfactorily with the value obtained from the reaction velocity data.

Again, in connection with solutions, especially solutions of electrolytes, another problem arises, namely, the possibility of applying the concept of energy of activation and the radiation hypothesis to the idea of activity or thermodynamic concentration, a term first introduced by G. N. Lewis. As is well known it is necessary to introduce activity in place of concentration in order to account for the experimental results obtained in connection with the electromotive force of concentration cells and the other properties of solutions thermodynamically inter-related, including the law of mass action.

The use of concentration at all in such physico-chemical considerations has its origin in the assumption made by Guldberg and Waage, according to which the concentration of a component is a measure of its active mass. It may be surmised that this assumption from its very simplicity is scarcely likely to be sufficiently comprehensive. Thus it tells us nothing of the effect of temperature or of material environment upon active mass although active mass varies with both. At the same time the success which has attended the application of the Guldberg-Waage hypothesis leads us to expect that the more general relation is to be sought in an extension of the Guldberg-Waage idea. From several points of view the active mass or activity $a$ appears to be related to the concentration $c$ by means of an expression such as :

$$
a=\mathrm{k}_{\mathrm{o}} c e^{-\mathrm{E} / \mathrm{RT}}
$$

where $k_{\mathrm{o}}$ is a proportionality factor, and $\mathrm{E}$ is the critical increment or energy of activation per grammolecule of the substance with respect to the chemical change contemplated. ${ }^{2}$ Such an expression makes the activity a function of the temperature, and, at the same time, since $E$ varies with the material environment (as has already been indicated in connection with reaction velocity data), the activity will likewise vary with the environment. At constant temperature and constant material environment (i.e. gaseous systems or very dilute solutions in one and the same solvent) the expression reduces to that of Guldberg and Waage. From the statistical point of view the exponential term gives the fraction of molecules in the chemically active state. The same result is obtained from the point of view of the quantum theory. ${ }^{3}$ Thus, in connection with the relation of emission to non-emission by an oscillator, Planck ${ }^{4}$ postulates that the ratio of the probability that no emission takes place to the probability that emission does take place is proportional to the radiation density $u_{v}$ of the frequency $v$. The ratio is given by $p u_{\nu}$, where $p$ is shown to be identical with $c^{3} / 8 \pi h n^{3} v^{3}, c$ being the

\footnotetext{
1 Loc. cit.

${ }^{2}$ For pure substances the activity is usually regarded as being measurable by the vapour pressure, which is an exponential function of the temperature of the same form as that employed above.

${ }^{3}$ Cf. Fourn. Chem. Soc., II3, 475 (I9I8).

"Theory of Heat Radiation," English ed., p. 164.
} 


\section{RADIATION HYPOTHESIS OF CHEMICAL REACTIVITY}

velocity of light in vacuo. Since $u_{\nu}$ itself is $\frac{8 \pi h n^{3} v^{3}}{c^{3}} e^{-h \nu / k \mathrm{~T}}$ (for the range of frequencies usually regarded as of significance for chemical change), the product $p u_{v}$ is simply $e^{-k v / k \mathrm{~T}}$ or $e^{-\mathrm{E} / \mathrm{RT}}$. So long as no emission of a given frequency takes place the energy of the oscillator, and therefore of the atom or molecule, remains high with respect to this type. Such a molecule will correspond to an active one in the chemical sense. We may apply Planck's postulate therefore to the chemical case thus :

$$
\frac{\text { number of chemically active molecules }}{\text { number of inactive molecules }}=e^{-\mathrm{E} / \mathrm{RT}}
$$

and since, in general, the number of inactive molecules is practically identical with the total number of molecules of the species, the above expression stands for the active fraction. Hence when the total concentration is $c$, the concentration of active individuals is $c e^{-\mathrm{E} / \mathrm{RT}}$. Consequently the suggestion made above amounts to saying that the active mass of a dissolved substance is proportional to the number of active molecules. present.

The variation in $\mathrm{E}$ with an alteration in the material environment in the case of ions is well illustrated by the following results obtained by the electromotive force method by Mr. T. Moran in the writer's laboratory using. a cell of the type: Hydrogen electrode, intermediate liquid, normal calomel electrode. The electrolyte, hydrochloric acid, is maintained throughout at constant concentration, namely decinormal. This means that the concentration of the hydrogen ion is either exactly or approximately constant. The variations in the observed activity-expressed as usual in gramequivalents per litre-are therefore to be ascribed almost entirely to an alteration in $\mathrm{E}$. The first table $\mathrm{A}$ shows the increase in the activity of hydrogen ion (owing to decrease in $\mathrm{E}$ ) due to replacement of water by sucrose. In table $\mathrm{B}$ a similar effect is shown owing to the presence of potassium chloride, and in table $\mathrm{C}$ the simultaneous effect of sucrose and $\mathrm{KCl}$.

TABLE A.

o.r $\mathrm{N} \mathrm{HCl}+$ Sucrose. Temp. $25^{\circ} \mathrm{C}$.

\begin{tabular}{|c|c|c|c|}
\hline $\begin{array}{l}\text { Concentration of } \\
\text { Sucrose in Grammole- } \\
\text { cules per Litre of } \\
\text { Solution. }\end{array}$ & $\begin{array}{c}\text { Concentration of } \\
\text { Water in Grammole- } \\
\text { cules per Litre. } \\
\mathbf{M}=18\end{array}$ & $\begin{array}{l}\text { Electromotive Force } \\
\text { of Cell in Volts. }\end{array}$ & $\begin{array}{c}\text { Activity of } \mathrm{H}^{+} \text {in } \\
\text { Gram-equivalents } \\
\text { per Litre. }\end{array}$ \\
\hline 0 & $55^{\prime} 55$ & 0.3475 & 0.080 \\
\hline 0.292 & $5 I \cdot 95$ & $0 \cdot 3428$ & 0.096 \\
\hline $0.5^{8} 5$ & $48 \cdot 45$ & $0 \cdot 3381$ & 0.155 \\
\hline 0.877 & $44^{\circ} 99$ & 0.3334 & O.I39 \\
\hline$r \cdot r 69$ & $4 x \cdot 62$ & 0.3287 & 0.167 \\
\hline$I \cdot 460$ & $3^{8 \cdot 09}$ & $0^{\prime} 324^{\circ}$ & 0.200 \\
\hline $\mathrm{I} \cdot 755$ & $34 \times 59$ & 0.3194 & 0.240 \\
\hline $2 \cdot 047$ & $30^{\circ} 94$ & 0.3147 & 0.288 \\
\hline
\end{tabular}

It will be observed that the activity of hydrogen ion can be raised from 0.080 to 0.288 , that is a threefold increase by substituting 70 per cent. sucrose solution for water. Similarly the presence of 3 normal $\mathrm{KCl}$ doubles the activity of the hydrogen ion, whilst the activity is increased fivefold by the simultaneous presence of 60 per cent. sucrose and ro per cent. $\mathrm{KCl}$. In the last case the observed increase in activity expressed in gram-equivalents per litre really means on the basis of the suggestion made earlier that 
TABLE B.

$0 \cdot \mathrm{I} \mathrm{HCl}+\mathrm{KCl}$.

\begin{tabular}{|c|c|c|c|}
\hline $\begin{array}{l}\mathrm{KCl} \text {, Grammolecules } \\
\text { per Litre. }\end{array}$ & $\begin{array}{c}\text { Concentration of } \\
\text { Water in Grammole- } \\
\text { cules per Litre. }\end{array}$ & $\begin{array}{l}\text { Electromotive Force } \\
\text { of Cell in Volts. }\end{array}$ & $\begin{array}{c}\text { Activity of } \mathrm{H}^{+} \text {in } \\
\text { Gram-equivalents } \\
\text { per Litre. }\end{array}$ \\
\hline 0 & $55 \cdot 55$ & 0.3475 & 0.080 \\
\hline $0.67 x$ & $54^{\circ} 29$ & 0.3449 & 0.089 \\
\hline$I \cdot 34^{I}$ & $53 \cdot 26$ & $0 \cdot 3407$ & 0.105 \\
\hline $2 \cdot 012$ & 5 I'96 & $0.335^{8}$ & 0.126 \\
\hline $2 \cdot 682$ & 50.83 & $0 \cdot 3307$ & $0 \cdot 154$ \\
\hline $3 \cdot 353$ & $49 \cdot 63$ & 0.3255 & $0 \cdot I 89$ \\
\hline
\end{tabular}

TABLE C.

O. I $\mathrm{N} \mathrm{HCl}+\mathrm{KCl}+$ Sucrose.

Concentration of $\mathrm{KCl}=\mathrm{I} \cdot 34 \mathrm{I}$ molar.

\begin{tabular}{|c|c|c|c|}
\hline $\begin{array}{l}\text { Concentration of } \\
\text { Sucrose in Grammole- } \\
\text { cules per Litre. }\end{array}$ & $\begin{array}{l}\text { Concentration of } \\
\text { Water in Grammole- } \\
\text { cules per Litre. }\end{array}$ & $\begin{array}{l}\text { Electromotive Force } \\
\text { of Cell in Volts. }\end{array}$ & $\begin{array}{c}\text { Activity of } \mathrm{H}^{+} \text {in } \\
\text { Gram-equivalents } \\
\text { per Litre. }\end{array}$ \\
\hline 0 & $53^{\cdot} 26$ & 0.3407 & $0^{\circ} \mathrm{IO}_{4}$ \\
\hline 0.292 & $49 \cdot 63$ & 0.3369 & $0.12 \mathrm{I}$ \\
\hline 0.585 & $46 \cdot 19$ & $0 \cdot 3320$ & 0.147 \\
\hline 0.877 & $4^{2 \cdot 64}$ & $0 \cdot 3262$ & 0.184 \\
\hline$I \cdot I 69$ & $39 * 18$ & 0.3200 & 0.234 \\
\hline$I \cdot 460$ & $35^{\circ} 4^{8}$ & $0.3 \times 30$ & 0.308 \\
\hline$I \cdot 755$ & $32^{\circ} \mathrm{Or}$ & $0 \cdot 3050$ & $0 \cdot 420$ \\
\hline
\end{tabular}

the critical increment $\mathrm{E}$ has diminished by $9^{8} \mathbf{I}$, or, in round numbers, I 000 cals. per gram-ion. This should be detectable ultimately by a spectroscopic method, by observing a shift in the position of a band in the infrared region. At present, owing to the numerous bands exhibited by water itself, it is not yet possible to say with certainty what band is to be attributed to hydrogen ion.

The radiation hypothesis has been criticised from three points of view. In the first place it has been criticised on the ground that the rate of supply of radiant energy of a given frequency and at the temperature of the matter is insufficient to account for the observed rate of the chemical process, even in the case of reactions carried out in an isothermal manner. This criticism is implicitly founded on the assumption, however, that the amount of radiation (of the frequency which the matter can absorb in the chemically active region) coming from the walls is the only source of supply. It overlooks the adjustment already referred to, whereby the effective supply is the whole range of frequencies present in virtue of the temperature of the system, conversion from one type to another taking place by reflection and absorption and emission between the oscillators present in the system itself and the material surroundings, the thermostat or constant temperature furnace. When this is taken into account, then, so far as amount of energy is concerned, the supply is more than sufficient. ${ }^{1}$

The second criticism is based on photo-chemical considerations, it being pointed out, for example, that the exposure of many systems to sunlight does not sensibly increase the observed velocity of the reaction, 


\section{RADIATION HYPO'THESIS OF CHEMICAL REACTIVITY}

although in sunlight itself the radiation density corresponding to any frequency is very much greater than the corresponding density in the material system maintained at ordinary temperature. Since, however, the matter and radiation are in this case not at the same temperature it is unjustifiable to compare the relative effects at all, for the following reasons. Owing to the lack of temperature equilibrium, when a substance is exposed to high temperature radiation, there is a tendency for the temperature of the matter to rise and that of the radiation to fall. This is brought about by absorption chiefly of a physical nature, i.e. unaccompanied by chemical change probably at numerous positions, such absorption occurring in a layer of moderate thickness, so that throughout the bulk of the system the radiation density is scarcely altered from its original value prior to illumination, and, consequently, the rate is not appreciably affected. This is particularly in evidence in the case of aqueous solutions, owing to the great absorption capacity of water in the short infra-red region, the region which contains the chemically effective frequency for the majority of reactions which can take place at a measurable rate in solution. Thus with a layer of water $0.12 \mathrm{~mm}$. in thickness the transmission at $\mathbf{I} \mu$ is 83 per cent., at $I^{\cdot} 5 \mu$ 45 per cent., at $2 \mu$ I 9 per cent., and at $3 \mu$, I per cent. ${ }^{1}$ Hence at $2 \mu$, for example, the energy of the incident light under photo-chemical conditions will fall to $10^{-6}$ of its initial value after traversing a thickness of I $\mathrm{mm}$. In the case of inversion of sucrose and similar reactions involving hydrolysis, although the total critical increment corresponds to a wave-length of the order $\mathrm{I}$ to $2 \mu$, it is almost certain, in virtue of the complex formation and the rupture of more than one bond, that each individual quantum involved does not exceed that corresponding to $3 \mu$ and still longer wave-lengths. At such positions the thickness required to reduce the intensity even of sunlight to that of the matter will be considerably less than $1 \mathrm{~mm} .^{2}$ Incidentally it may be mentioned that unpublished measurements by H. A. Taylor in the writer's laboratory have shown that the determination, with a reasonable degree of precision, of the absorption coefficient of a liquid in the short infra-red region is one of very great difficulty owing to the large heating effect, due to the large physical absorption, which occurs in an extremely thin layer on the exposed side of the liquid. In fact, the only satisfactory mode of working is to renew the liquid rapidly. Obviously the same heating effect must enter into attempts made to determine possible photo-chemical acceleration in this region, so that even where a slight acceleration has actually been observed it has not yet been possible, in the writer's opinion, to distinguish in the case of liquid systems the effect due to local heating from a true photo-effect, if, in fact, such a distinction can be made at all in a region in which the solvent itself absorbs strongly. In such a case the solvent acts as a protective screen for the solute, so far as direct photo-chemical effects are concerned, at the same time greatly increasing the thermal decomposition of the solute in a very thin layer owing to local rise in temperature. The net result for a solution in bulk must, however, be very small. It is necessary to emphasise the fact that the existence of an absorption coefficient characterises a photo-chemical process and thus distinguishes photo-chemical conditions from thermal. Its existence indicates lack of equilibrium between the radiation and the matter and the tendency of both to arrive at the same temperature.

The third criticism of the radiation hypothesis is that in certain cases, notably in gaseous reactions, at the position which corresponds to the

${ }^{1}$ Cf. Coblentz, Carnegie Inst. Pub., No. 35, p. I30 (I905).

${ }^{2} C f$., however, Taylor, Nature, Oct. I3 $_{3}$ p. 2 Io; and Lewis, ib., Oct. 20, p. 24I (I92I). 
critical increment of the process the substance possesses no absorption band when examined spectroscopically, i.e. photo-chemically, and consequently is incapable of responding to radiation of this frequency under thermal conditions. This criticism appears to be justified and to involve therefore a certain modification of the radiation hypothesis, a modification already hinted at, namely, absorption at a frequency which is a submultiple of that calculated from the critical increment. The calculation of the active frequency or wave-length from the kinetics of the chemical process is based on the simplest possible assumption, namely, that one quantum of the given frequency decomposes or activates one molecule to the extent required for the process. This is in fact Einstein's law of the photochemical equivalent. In view of the criticism just referred to and also in view of the facts mentioned below it would seem necessary to conclude that in certain cases a limited number of quanta of smaller magnitude take the place of the single quantum, the wave-length, corresponding to the smaller quanta, occurring naturally further towards the infra-red.

On the basis of Planck's theory as the energy content of an oscillator increases, so as to approach one quantum of the characteristic frequency, a state of instability is produced in the oscillator, as a result of which emission of radiation may occur. If no such emission takes place the energy content of the oscillator increases further until the region of two quanta is approached at which a further opportunity for emission occurs. As the energy content rises in this manner the probability of an oscillator attaining to a large number of quanta becomes rapidly smaller. It follows that in general only small numbers of quanta, that is numbers not greatly removed from unity, may be considered as of significance. Probably the multiple does not exceed four or five and may be even less. This view, in its limitation (on Planck's basis) to the number of subquanta which can be held, differs from that recently put forward by Professor Baly, ${ }^{1}$ according to which the transference of energy takes place in the long infra-red region, ${ }^{2}$ which presumably means that a considerable number of such small quanta have to be assumed to be absorbed in order to attain the magnitude required for the chemical change.

The first apparently indubitable evidence that the absorption of energy does not necessarily take place in one quantum - though this does occur in other cases-is that obtained by Daniels and Johnston ${ }^{3}$ on the decomposition of nitrogen pentoxide. Taking the critical increment of the reaction, as do the authors themselves, to be 24,700 cals., the corresponding wavelength is $1 \cdot 16 \mu$. Taking the critical increment on the basis of Daniel and Johnston's data to be $25,130,{ }^{4}$ the corresponding wave-length is $I^{\prime} I 3 \mu$. Daniels and Johnston have found that nitrogen pentoxide is not decomposed by radiation in the region of either of these almost identical values. Hence the thermal process does not occur through one quantum of this frequency. Daniels and Johnston point out that $\mathrm{I} \cdot \mathrm{x} 6 \mu$ corresponds to a frequency exactly five times the frequency of the most marked band in the infra-red spectrum of $\mathrm{N}_{2} \mathrm{O}_{5}$, namely, at $\lambda=5.8 \mathrm{I} \mu$, observed by Warburg and Leithauser, ${ }^{5}$ and though they do not explicitly connect the two observations it is fairly evident that they consider the possibility of making up the energy required for the reaction from five of such subquanta. It will be observed that we are here dealing with a relatively small multiple,

\footnotetext{
${ }^{1}$ Baly and Barker, Fourn. Chem. Soc., r r9, 653 (rgar).

5 Ann. Physik [IV.], 28, 3r3 (rgog).
} 


\section{$5^{86}$ RADIATION HYPOTHESIS OF CHEMICAL REACTIVITY}

namely five. It is more probable, however, that an even smaller multiple is involved. Thus nitrogen pentoxide has several bands in the short infrared, the band at $3.39 \mu$ being second in order of intensity. It will be observed that this is exactly three times the wave-length $I^{\cdot} 13 \mu$ calculated as a possible position from Daniel and Johnston's data, so that the significant multiple in this case appears to be three.

A similar consideration must apparently be taken into account in dealing with the dissociation of hydrogen and of phosphine. The heat of dissociation of hydrogen as determined by Dr. Langmuir, together with the assumption made by the writer that free atoms do not require any activation in respect of recombination, leads us to expect that hydrogen should exhibit an absorption band in the neighbourhood of $330 \mu \mu$, provided the energy is absorbed in one quantum. Mr. L. W. Codd, in the writer's laboratory, has examined hydrogen very carefully in the visible and ultra-violet regions, the gas being kept at a temperature of approximatèly $400^{\circ} \mathrm{C}$. and at a pressure of six atmospheres. Even under these conditions no trace of absorption manifested itself. It cannot be asserted of course that absorption would be absent at still higher pressures, but in the present state of affairs it seems inevitable to assume that the dissociation is brought about by energy absorbed at a longer wave-length. By analogy with nitrogen pentoxide, if we take 3 as the significant multiple, we would conclude that the active region might be in the neighbourhood of $990 \mu \mu$. No direct experimental data are as yet available in this connection. It may perhaps be mentioned that Abney and Festing, ${ }^{1}$ who examined by a photographic method a number of organic substances, concluded that the absorption in this region was due to the presence of hydrogen in the molecule. A more suggestive result was obtained by Liveing and Dewar, ${ }^{2}$ namely, a reversal of the $\mathrm{F}$ and $\mathrm{C}$ lines of hydrogen. They state that under certain pressure conditions the $F$ line becomes a band. The wave-length of this, namely, $487 \mu \mu$, is approximately two-thirds of that anticipated for the dissociation of hydrogen on the single quantum basis.

In the case of phosphine apparently a similar state of affairs exists. Codd observed no absorption at all through a layer of gas of approximately six metres in thickness at room temperature until the region $260 \mu \mu$, at which absorption commences and is still persisting at 2 I $0 \mu \mu$, the limit set by the plate employed. This region, however, is well beyond that corresponding to the decomposition of phosphine, namely $375 \mu \mu$, on the single quantum basis. In Codd's experiments the phosphine was decomposed.

In contradistinction to the behaviour of $\mathrm{N}_{2} \mathrm{O}_{5}, \mathrm{H}_{2}$, and $\mathrm{PH}_{3}$, the evidence obtained by Lyman in connection with the formation of ozone points very strongly to the conclusion that the dissociation of oxygen can only be brought about (in the homogeneous gaseous state) by very short wave-lengths, i.e. in the region of $185 \mu \mu$, and presumably, therefore, by one quantum of such radiation.

A further point may be alluded to. Physically there is a clear distinction between the continuous absorption of radiant energy until a single quantum of relatively high grade type has been accumulated and the absorption of the same amount of energy of relatively low grade type, in terms of a limited number of quanta of the latter type. In spite of this physical distinction, however, the final expressions obtained for the velocity constant of a unimolecular process are very similar and of the same order of magnitude. It may be shown that in the case of the multiple 3 (i.e.

${ }^{1}$ Phil. Trans. Roy. Soc., I72, 887 (r881). I Proc. Roy. Soc., 35, 74 (1883). 
absorption at a wave-length one quantum of which is one-third of the total energy actually required), the velocity constant is one-ninth of that calculated on the single quantum basis. In fact the calculated velocity constant is $I / p^{2}$ of that calculated on the single quantum basis, where $p$ is the multiple.

On reviewing the problems presented by chemical kinetics in homogeneous systems, both liquid and gaseous, one is led to regard the radiation hypothesis as in general a useful guide in the further elucidation of such problems.

\author{
Muspratt Laboratory of Physical \\ and Electro-Chemistry. \\ University of Liverpool.
}

